\title{
Research on E-commerce Enterprise Management Based on Big Data
}

\author{
Yuanjian Qin ${ }^{a}$, Yuming Zhao ${ }^{\mathrm{b}}$ \\ Schools of Management, Wuhan University of Technology, Wuhan 430070, China.

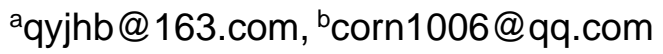

Keywords: Big data, e-commerce, enterprise management.

\begin{abstract}
Along with the popularization of the Internet and the arrival of big data era, every field in daily life has been strongly impacted and influenced. The application of big data based on Internet technology has greatly changed people's production and consumption, the influence is very far-reaching. The continuous increase of data volume on the Internet has virtually changed people's ideas and behaviors. The mining and development of big data are effective technological means to promote the transformation and development of e-commerce enterprise; it also brings severe challenges to the operation and development of e-commerce enterprises. Modern enterprise management is also developed from the traditional "business-driven" to the direction of "data-driven", the analysis and use of big data can provide reliable and solid guarantees for business management and decision-making, and formulate more scientific and reasonable management models. However, when studying the management status of e-commerce enterprises under the background of big data, it was found that there are some problems need to be solved in the e-commerce enterprise management. Based on this point, this paper takes the e-commerce enterprise management model as the research content under the background of big data era, give a brief overview of the meaning of big data, and discuss e-commerce enterprises under the background of big data era, and then analyze the management mode of the e-commerce enterprise under the background of big data era, so provide certain reference for the management of e-commerce enterprises and propose reasonable improvement measures.
\end{abstract}

\section{Introduction}

The development of Internet technology has provided huge opportunities for e-commerce enterprise; the application of big data based on Internet technology has provided a broader platform for the development of e-commerce, e-commerce enterprises is flourishing, the number and or scale of enterprises all show a straight rising trend, it promoted the development of e-commerce market to a certain extent. However, with the development of the times, the management mode of e-commerce enterprises also needs timely improvement and adjustment, and then adapts to the development requirements of big data era. At present, there are still many loopholes and drawbacks in the current management model of e-commerce enterprises, many places still need to be corrected, under the background of big data era, e-commerce enterprises should also pay attention to various challenges while facing opportunities. The e-commerce enterprises should actively participate in them in the face of the challenges and opportunities brought by big data, introduce advanced management technologies and management concepts, promote management model innovation, and strengthen research on e-commerce enterprise management models, which will help improve defects and deficiencies of traditional management models, enhance the competitiveness of enterprises, and seek long-term survival and development.

\section{Big Data}

\subsection{Meaning of Big Data.}

Among the various types of data, the technology which can effectively screen and rapidly find valuable data is called as big data technology. Big data is a concept relative to current data storage, computation and processing mode, it is a more comprehensive and overall concept, including 
technological and commercial aspects, it can effectively screen each type of data quality and quickly find valuable technological data. Big data technology is a bridge that quickly obtains valuable information from much diverse data, collects big data at high speed to discover its characteristics and acquire new technologies.

Under normal circumstances, big data is a new type of technological framework that rapidly analyzes data in large amounts of data and discovers its characteristics and gain value through high-speed capture. The characteristics of big data are clear, the total amount of data operations is large, the data is running fast, and it has various and accurate features in operation. Big data is highly efficient in predicting information and can accurately analyze the correlation among the data. In the past, data analysis was only the sample survey of the data, and it was only able to get causality but could not acquire the correlation. The essence of big data is based on a relational relationship, and the data is predicted by relational analysis.

Nowadays, big data is used to generate mass structured or unstructured data in non-traditional IT equipment channels such as Internet, mobile, medical treatment and video monitoring, at the same time, modern management and operation are affected, the enterprises obtain effective information about consumers from the transaction records of data, can better understand the actual situation of suppliers and operators; sensors are implanted into mobile devices, the transaction data can be obtained from the meter, cell phone, car and so on, so as to realize the inductive exchange of data.

\subsection{E-commerce in the Era of Big Data.}

The birth of big data has brought great commercial value; especially application value aspect is inestimable, the development of big data has affected all aspects of society. The emergence of big data has promoted innovation, increased labor productivity, achieved new economic growth, and created new competitive models and business value. Up to this day, e-commerce has become a fast-growing, high-value industry.

The arrival and rapid development of the big data era has brought unprecedented opportunities for e-commerce retail enterprises, many e-commerce enterprises have sprung up, and the arrival of big data has led to the emergence of retail e-commerce enterprises, and the emergence and development of e-commerce enterprises accelerate the use of big data. This is mainly because the popularity of the Internet and the development of related technologies make it possible to collect, manage, and analyze mass data, the Internet make these data to be transmitted at high speeds and volumes. At the same time, the data introduced by the Internet is generated by users; these data are characterized by multiple sources, low cost, which provide important conditions for the rapid development of e-commerce enterprises. One of the advantages of retail e-commerce is the availability of various data and to traditional retail industry; e-commerce enterprises can obtain the visit source of customers in real time.

For example, through the search, purchase, collection, and purchase of goods within the website, all can provide real-time data for e-commerce, these data can help e-commerce enterprises to serve customers more effectively and accurately, change original marketing management model e-commerce, and promote innovation of service management model. Providing corresponding personalized services for the consumers' characteristics is an important trend and revolution in big data service. This is because the huge consumer data provides e-commerce enterprises with the foundation to grasp users' consumption, e-commerce enterprises can effectively implement personalized, accurate, and intelligent ad-pushing services through big data applications, create a more high-efficiency marketing and management model, so that the actual application of data can be more organically integrated with the enterprises' operation.

\section{Analysis of the Management Mode of E-commerce Enterprises under the Background of Big Data Era}

\subsection{Low Cost Management.}

At this stage the information service and data processing modes are different from the traditional ones, traditional information processing modes are tedious and complex, and the efficiency and 
accuracy of information processing are much lower. The big data technology is used for information processing is quite convenient and quick. In a very short period of time, we can collect, process, handle, analyze mass data and calculate accurate results. Many large enterprises can collect and handle customers' information in a fully automated manner, automated systems are extensively used for a large number of customer data, and then achieve collection, arrangement and analysis of information, this will enable enterprises to effectively understand and grasp the true state of their customers, and provides strong data support for the future development of enterprises. Throughout the entire process, due to the reduction of manual intervention of links, the business level and work efficiency have been greatly improved, the error rate has been significantly reduced, and the cost of data processing has been reduced to a certain extent, it is also provides a new mode of thinking for innovative data management of e-commerce enterprises.

\subsection{Fine management.}

The traditional business model is difficult to achieve process all kinds of data in large numbers, it has caused many enterprises can only analyze and store data by extracting a small number of samples in the traditional management mode, and analyze the corresponding results, so further infer the overall characteristics. Such traditional technology methods is a great waste of human and material resources, and it can only record customers' simple consumption information of clients, cannot fully analyze and handle the customers' personal information, the results of such management cannot achieve fine management. Under the background of big data era, e-commerce enterprises have a very powerful ability to process data; they can accurately extract information from each customer, and then through corresponding analysis and processing, collect and summarize the data of customers' consumption situation and so on, use data mining technology for effective processing and analysis, and finally form an effective management. This method is convenient, fast, and cost-effective, the most important thing is that the utilization and value of data have been greatly improved, make enterprises achieve fine management to a certain extent. So that e-commerce enterprises have a broader development space.

\section{Development Directions and Challenges of E-Commerce under Big Data Environment}

\subsection{Development of E-commerce Based on Big Data.}

The main development direction of big data is a new generation of innovation for data service; it is to provide a personalized product for every group in the society and even each individual, thus gradually forming a personalized service. A large number of e-commerce transactions provide e-commerce enterprises with enough network consumption data, through accurate data analysis, the consumers and bias of consumer are planned, and these provide the basis for business model changes.

With the development of electronic commerce, the information content has developed rapidly; it make consumers difficult to choose in the face of a large amount of information data, at the same time, the screening analysis of product information has not been improved, which make it impossible for consumers to accurately grasp the latest dynamic information. For this purpose, the future development direction of e-commerce lies in providing consumers with accurate service information and forming a personalized service concept. The development of big data provides data foundation for e-commerce enterprises to develop personalized service. Through the analysis of big data and the use of information brought by big data, enterprises can find potential customers and effectively communicate with customers during the sales process, so obtain the potential profit value in marketing. In this way, enterprises can quickly occupy the market, save costs, achieve high-efficiency sales performance, thus increasing competitiveness and overcome opponents.

\subsection{The Challenge of E-Commerce under Big Data Environment.}

The arrival of the big data era has become the basis for the innovation of e-commerce enterprises and it is important opportunity for its development. How to master big data and conduct security and privacy management of data is an important issue that e-commerce enterprises have to face.

Mastering big data is one of the contents that rely on big data development. Under normal circumstances, the production of big data is mainly through three aspects. The first is obtained 
through the search results of web search engines, including the click number of advertisements, search information of enterprise websites, link data, etc., thus summarizing data; secondly, data generated by users on mobile devices, such as mobile phone, computers and other devices directly access the platform's data, as well as the use of the browser and the mobile device's own application software; finally, integrate the data of some shopping websites and other online trading platforms. If e-commerce enterprises develop personalized user services, they must master big data, and the premise is to obtain data. For small and medium-sized e-commerce enterprises that want to develop precise services, it is necessary to solve this important problem of mastering big data, and make reasonable and effective planning for the future development of the enterprises.

In the era of big data, each network user's behavior record will be collected by e-commerce enterprises, casual speech, browsing of images and videos on the Internet and registered personal information will be recorded in the database. By arranging these data, e-commerce enterprises have mastered each user's personal orientation, and analyze and predict based on this, thus exploiting potential business value, create new business projects, and improve profit margins. The information of the network users usually contains the user's real information and related financial card numbers and other privacies, once leaked, the users will be troubled, this problem is the problem that the e-commerce enterprises must solve. How to ensure the security of data and user privacy is a major challenge for e-commerce enterprises, enterprises must set up multiple levels of protection measures, highly value data security can prevent disputes caused by user privacy leakage, and reduce the legal troubles caused by the enterprises' secret leakage.

\section{Improvement Measures of E-commerce Enterprise Management under Big Data Environment}

\subsection{Precision Marketing and Establish Customers' Large Database.}

Precision marketing mainly uses modern information technology to analyze and process data information in depth, communicates and exchanges with customers, and constantly adjusts strategic deployment, provide more targeted services for customers, and improve service quality, adjust marketing and management modes of enterprises. Through precision marketing, e-commerce enterprises can more fully understand consumers' consumption situation and purchasing power, and provide practical solutions for enterprises' development. Through the analysis of customer purchasing behavior and trends, collect customers' hobbies, analyze the customer's consumption level, build large database of customers, through modern data mining technology analysis and forecast, provide customers with more targeted products.

\subsection{Innovate Management Concept.}

Under the background of big data, the traditional business model is mainly based on data sampling analysis, and further judges the overall performance based on the analysis results, this model can no longer meet the processing needs of massive data, data analysis and storage need to be implemented with more advanced big data technologies. At the same time, the customers' information is extracted accurately, grasps customers' consumption habits and trends, use data mining technology to analyze systematically, improves information processing efficiency, reduces costs, achieves precision management, and promote customers to enjoy personalized and intelligent services, and promote the development of e-commerce enterprises. For example, production-oriented enterprises can implement precision management for products stock of enterprises according to the results of information processing, through analysis of data; the problems existing in the current enterprises' stock can be analyzed, provide real-time stock monitoring, and timely handle it, and achieve precision management.

\subsection{Collect Customers' Data and Cut Management Cost.}

Compared with traditional information processing methods, the processing flow of data information is further simplified under the background of big data; business processing is low cost and high efficiency, rapid collection and processing of information are achieved through big data technology the results are more accurate. In particular, many e-commerce enterprises automatically 
collect users' data information through automated means, they have full understanding for the customers' consumption situation and hobbies, which help the adjustment of the enterprises' business strategy, reduce the probability of human intervention as much as possible, effectively improve business efficiency, and it has profound significance for the innovation of e-commerce enterprises' management model.

\section{Conclusion}

To sum up, this paper analyzes and studies the management model of e-commerce enterprises under the background of big data era, the e-commerce management model has dynamic characteristics, and it will change with time, therefore, if enterprises only rely on imitation instead of independent innovation management, their development will be greatly limited. E-commerce enterprises should further promote the innovation and improvement of management models, give full play to the systematic analysis of big data technologies, provide personalized and intelligent services for customers, enhance the core competitiveness of enterprises, and create greater economic benefits for enterprises.

\section{References}

[1]. Liu Zhuhai, Chen Yong, Yao Chili. Innovation of E-business Service Model in Big Data Era [J], Science and Technology Management Research, 2014(1).

[2]. Gann Lexan, To Wei. Discussion about Opportunities and Challenges of E-Commerce in Big Data Era [J], Science Mosaic, 2013(03).

[3]. Ren Guangjian. Business Model Innovation Based on "Big Data" and it’s Enlightenment [J], Modern Business Trade Industry, 2014(2).

[4]. Tina Xiaoping. Research on the Strategies of Enterprise Management Mode Innovation under the Background of Big Data Era [J], Value Engineering, 2016, (13):47-49.

[5]. Liu Jing. Analysis of the Management Modes of E-commerce Enterprises in the Era of Big Data [J]. Journal of Commercial Economics, 2017, (05): 99-101.

[6]. Zhang Cupping. Innovative Modes of Modern Enterprise Management in the Era of Big Data [J]. Market Modernization, 2015, (28): 83-84. 